\title{
A Detection of the Integrated Sachs-Wolfe Effect
}

\author{
S.P. Boughn ${ }^{\mathrm{a}}$ and R.G. Crittenden ${ }^{\mathrm{b}}$ \\ a Department of Astronomy, Haverford College, Haverford, PA 19041 \\ Department of Physics, Princeton University, Princeton, NJ 08544 \\ b Institute of Cosmology and Gravitation, University of Portsmouth, Portsmouth PO1 $2 E G$ UK
}

\begin{abstract}
We have detected statistically significant correlations between the cosmic microwave background and two tracers of large-scale structure, the HEAO1 A2 full sky hard X-ray map (Boldt, 1987) and the NVSS $1.4 \mathrm{GHz}$, nearly full sky radio galaxy survey (Condon et al., 1998). The level of correlations in these maps is consistent with that predicted for the integrated Sachs-Wolfe (ISW) effect in the context of a $\Lambda C D M$ cosmological model and, therefore, provides independent evidence for a cosmological constant. A maximum likelihood fit to the amplitude of the ISW effect relative to the predicted value is $1.13 \pm 0.35$ (statistical error only).
\end{abstract}

Key words: cosmic microwave background, X-rays: general, large-scale structure of the universe PACS: 98.80.Es, 95.85.Nv, 98.70.Vc, 98.65-r

In the currently favored $\Lambda$-cold dark matter $(\Lambda C D M)$ cosmological model, the dominant energy content of the universe is due to a cosmological constant, $\Lambda$, or some other form of "dark energy". The primary evidence for this model comes from supernovae redshift/magnitude observations (e.g. Barris et al., 2003) that imply the expansion of the universe is accelerating and from the spatial power spectrum of the fluctuations in the cosmic microwave background (Bennett et al., 2003). One of the consequences of this model is that a significant portion of the anisotropy of the $C M B$ is produced recently via a mechanism known as the integrated Sachs-Wolfe (ISW) effect (Sachs \& Wolfe, 1967). This anisotropy is created as CMB photons traverse the evolving gravitational potentials of linear density perturbations (i.e., $\delta \rho / \rho \ll 1)$ at relatively low redshifts $(z \lesssim 1)$.
Crittenden and Turok (1996) suggested that the ISW effect could be detected by correlating the CMB with some nearby $(z \lesssim 1)$ tracer of matter, e.g., galaxies or AGN.

A significant ISW effect arises recently in a curvature dominated universe or in a flat universe if it is $\Lambda$ dominated. On the other hand, in a flat, matter dominated universe the gravitational potentials of linearly collapsing structures are constant and there is no ISW effect. It is in this sense that the ISW effect has a unique sensitivity to a cosmological constant in a flat universe. If there is a significant $\Lambda$, then the CMB will be correlated with tracers of mass at low redshift; if not, there is no such correlation. To be sure, such correlations could be generated at roughly the same level if the universe is significantly open with $\Omega \lesssim 0.5$; however, the position of the Doppler peaks in the 


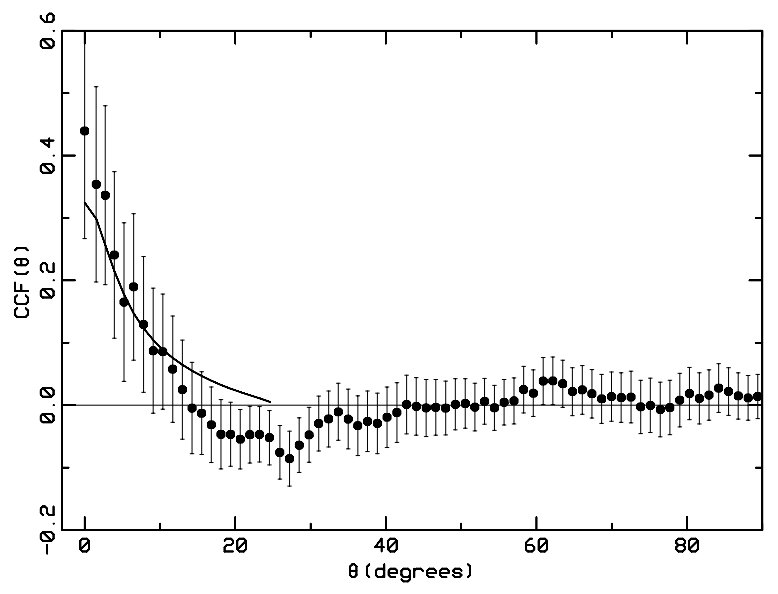

Fig. 1. The cross-correlation function of the WMAP ILC CMB maps with the HEAO1 A2 2-10 keV, hard X-ray map. The error bars were determined from Monte Carlo calculations and are highly correlated. The solid curve is the predicted ISW effect from the standard $\Lambda C D M$ cosmological model and is not a fit to the data. The units are $\mu K$ TOT counts $s^{-1}$ where 1 TOT count $s^{-1} \sim 1 \times 10^{-5} \mathrm{erg} \mathrm{s}^{-1} \mathrm{~cm}^{-2} \mathrm{sr}^{-1}$.

CMB anisotropy spectrum measured by WMAP and other experiments indicate that this is not the case (Bennett et al., 2003).

We have cross-correlated two mass tracers with the WMAP 'internal linear combination' (ILC) $\mathrm{CMB}$ map and found significant correlations in both cases (Boughn \& Crittenden, 2004a). The $H E A O 1 \quad A 2 \quad 2-10 \mathrm{keV}$ all sky X-ray map provides a good tracer of mass with a median redshift of about $z \sim 0.9$ (Boughn \& Crittenden, 2004b). Likewise, the NVSS $1.4 \mathrm{GHz}$ nearly full sky $(82 \%)$ radio catalog also provides a good tracer with the same median redshift (Condon et al., 1998). Both of these maps have significant systematics which we have tried to identify and correct for (Boughn \& Crittenden, 2002 Boughn. Crittenden \& Koehrsen, 2003). However, even if there are still residual contaminants, it is unlikely that the systematics in any of the three disparate data sets are correlated. In fact, the resulting cross-correlation functions are largely independent of the corrections.

A standard measure of the correlation of two data sets is the cross-correlation function $(C C F)$, which in this case is defined by

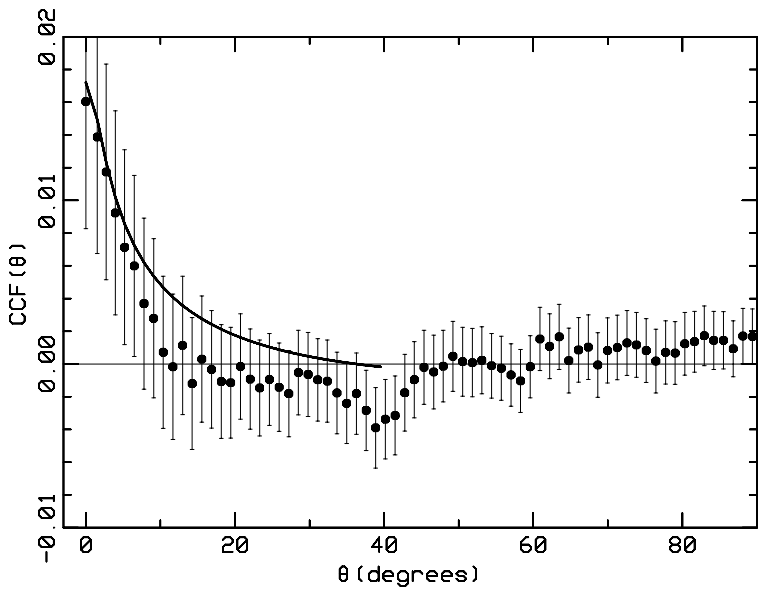

Fig. 2. The cross-correlation function of the WMAP ILC CMB maps with the NVSS $1.4 \mathrm{GHz}$ radio survey. The error bars were determined from Monte Carlo calculations and are highly correlated. The solid curve is the predicted ISW effect from the standard $\Lambda C D M$ cosmological model and is not a fit to the data. The units are $m K$ counts where the counts are number of radio sources per $1.3 \times 1.3$ degree pixel.

$C C F(\theta)=\frac{1}{N_{\theta}} \sum_{i, j}\left(S_{i}-\bar{S}\right)\left(T_{j}-\bar{T}\right)$

where the sum is over all pairs of pixels separated by an angle $\theta, S_{i}$ is the signal strength of the $i^{t h}$ pixel of the tracer map, $\bar{S}$ is the mean signal, $T_{i}$ is the CMB temperature of the $i^{t h}$ pixel, $\bar{T}$ is the mean CMB temperature, and $N_{\theta}$ is the number of pairs of pixels separated by $\theta$. In the case of the $H E A O \mathrm{X}$-ray map, $S$ is the $2-10 \mathrm{keV} \mathrm{X}$-ray intensity and for the $N V S S$ radio catalog, $S$ is the surface density of radio sources. Figure 1 is the X-ray $C C F$ and Figure 2 is the NVSS CCF. In both cases, the error bars were computed from Monte Carlo simulations and are highly correlated. These errors are primarily due to the fluctuations inherent in the X-ray, radio, and CMB backgrounds with only minor contributions due instrument noise and this is why they are so highly correlated. Photon shot noise in the X-ray map and Poisson noise due to finite source counts are important and account for roughly a half of the error.

The solid curves in the two figures are predictions of the currently favored $\Lambda C D M$ cosmological model and are not fits to the data. The statistical significance of the detection of the correla- 
tion is roughly $2.5 \sigma$ in both cases and it is clear that the correlation is consistent with the predicted ISW effect. Recall that in a flat, matter dominated universe, there is no expected correlation. A maximum likelihood fit to both data sets, taking into account that the two tracer maps are themselves correlated with each other, yields an ISW amplitude of $1.13 \pm 0.35$ relative to the predicted amplitude for the WMAP best fit cosmological model. This implies a $3.2 \sigma$ detection of the ISW effect with an amplitude consistent with that predicted by the standard $\Lambda C D M$ cosmological model.

The predicted ISW effect is sensitive to the biases of the two tracer maps, the redshift distribution of the sources, and to the the cosmological model. The redshift distributions were taken from model luminosity functions (Dunlop \& Peacock, 1990; Cowie et al., 2003) and the biases were determined from the the clustering of the sources in the two tracer maps (Boughn \& Crittenden, 2002, 2004b), the source redshift distributions, and the standard cosmological model. Even though there is considerable uncertainty in the luminosity functions and concomitant source redshift distributions, the predicted ISW effect is relatively insensitive to these uncertainties. For example, if the redshift distribution is artificially low, so will be the implied bias. However, in a $\Lambda C D M$ universe at these redshifts, the intrinsic ISW effect is somewhat larger at low redshifts and these two errors tend to cancel each other. For this reason, we conclude that this type of systematic error will be considerably smaller than the statistical errors indicated in Figures 1 and 2.

Another possible systematic error in the case of the X-ray/CMB analysis is nearby, unresolved source contamination if such sources emit significantly in both X-rays and microwaves. We masked the CMB map with the most aggressive WMAP mask $(k 0)$ that includes both the plane of the Galaxy and high latitude sources. The Galactic plane was also removed from the X-ray and, in addition, we masked high Galactic latitude X-ray sources in a variety of ways. However, the X-ray CCF is largely independent of how these sources are masked.

The NVSS/CMB CCF is less sensitive to high Galactic latitude sources since it is based on ra-

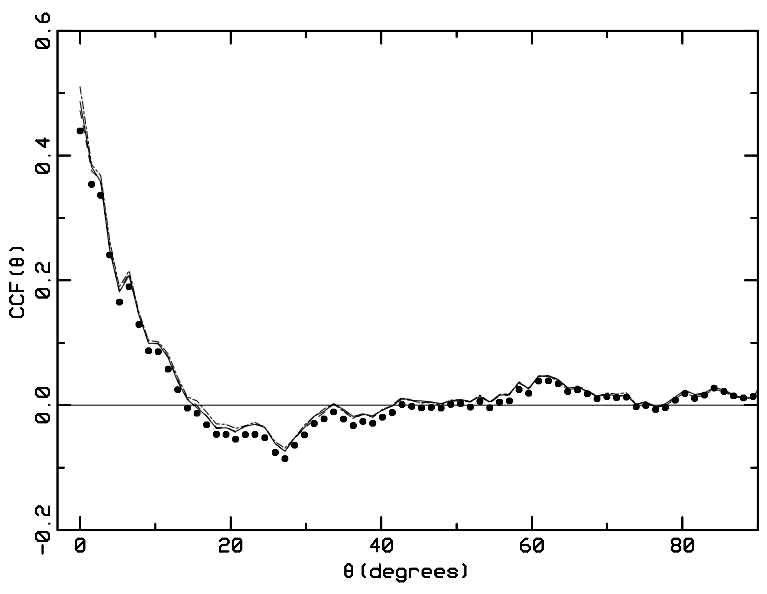

Fig. 3. The cross-correlation function of the $H E A O \mathrm{X}$-ray map with the 41 (solid curve), 61 (dashed curve) and 94 GHz (dot-dashed curve) WMAP CMB maps. The points are the cross-correlation with the internal linear combination map plotted in Figure 1.

dio number counts rather than source flux. More insidious is microwave emission from distant $\mathrm{X}$ ray and radio sources since it is these sources that are purported to map the mass distribution that generates the ISW signal. Models of AGN and radio sources indicate that they are not a source of contamination but an even stronger constraint can be obtained from the different WMAP CMB frequency bands $(41,61$, and $94 G H z)$ maps. Most radio/microwave sources have spectral indices, $\alpha$, ranging from $\alpha \simeq-0.7$ for a synchrotron spectrum to $\alpha \simeq-0.1$ for a Bremsstrahlung spectrum. On the other hand, the blackbody spectral index of the CMB is $\alpha \simeq+2.0$ in the Rayleigh-Jeans part of the spectrum. If the CCF we observe were due to radio source contamination then one would expect the CCF with the $94 \mathrm{GHz}$ WMAP map to be from 5.7 to 9.4 times larger than the CCF with the $41 \mathrm{GHz}$ map. Even inverted spectrum sources with spectral indices as large as $\alpha \simeq+1.2$ would imply a factor of two difference between these two CCFs.

Figures 3 and 4 are CCFs of the X-ray and NVSS maps with the 41, 61, and $94 \mathrm{GHz}$ CMB maps. (These three maps were corrected for Galactic emission using the synchrotron, free-free, and dust maps from the WMAP data public data set.) The solid and two dashed lines in the figures in- 


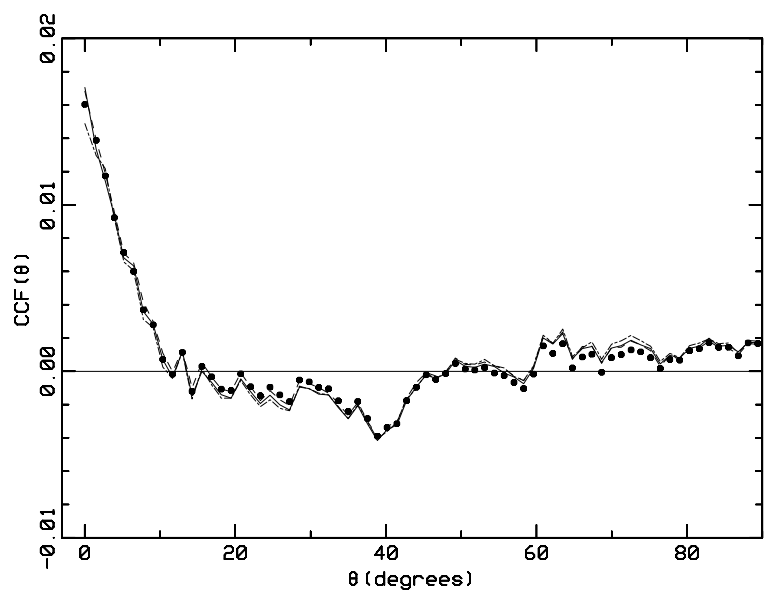

Fig. 4. The cross-correlation function of the NVSS radio map with the 41 (solid curve), 61 (dashed curve) and 94 $\mathrm{GHz}$ (dot-dashed curve) WMAP CMB maps. The points are the same as those plotted in Figure 2.

dicate the three CCFs while the points are the CCFs from Figures 1 and 2. It is clear that the difference between them is a few percent at most and we conclude that it is extremely unlikely that the observed correlation is due to radio source contamination.

The correlation functions of Figures 1 and 2 are consistent with the ISW effect predicted in a universe dominated by a cosmological constant, $\Lambda \sim$ 0.7 and, in this sense, provide important independent confirmation of the currently favored $\Lambda C D M$ cosmological model.

We are grateful to M. Nolta, L. Page and the rest of the WMAP team as well as N. Turok. We acknowledge the use of the Legacy Archive for Microwave Background Data Analysis (LAMBDA). Support for LAMBDA is provided by the NASA Office of Space Science

References

Barris, B. J. et al. 2003, ApJ, 598, 102.

Bennett, C. L. et al. 2003, ApJ Supp., 148,1.

Boldt, E. 1987, Phys. Rep., 146, 215.

Boughn, S. \& Crittenden, R. 2004a, Nature, 427, 45.
Boughn, S. \& Crittenden, R. 2004b, submitted to Apj, astro-ph/0404348

Boughn, S. \& Crittenden, R. 2002, PRL 88, 1302.

Boughn, S., Crittenden, R. \& Koehrsen, G. 2003, ApJ, 580, 672.

Boughn, S., Crittenden, R. \& Turok, N. 1998, New Astron., 3, 275.

Condon, J. et al. 1998, Astron. J., 115, 1693.

Crittenden, R. \& Turok, N. 1996, PRL 76, 575.

Cowie, et al. 2003, ApJ, 584, L57.

Dunlop, J. \& Peacock, J. 1990, MNRAS, 247, 19.

Sachs, R. K. \& Wolfe, A. M. 1967, ApJ, 147, 73. 